\title{
The SOX2 response program in glioblastoma multiforme: an integrated ChIP-seq, expression microarray, and microRNA analysis
}

Xuefeng Fang 1,2,3,5, Jae-Geun Yoon ${ }^{1}$, Lisha Li², Wei Yu², Jiaofang Shao ${ }^{2}$, Dasong Hua², Shu Zheng ${ }^{3}$, Leroy Hood ${ }^{6}$, David R Goodlett ${ }^{5}$, Gregory Foltz ${ }^{1 *}$, Biaoyang Lin ${ }^{1,2,4^{*}}$

\begin{abstract}
Background: SOX2 is a key gene implicated in maintaining the stemness of embryonic and adult stem cells. SOX2 appears to re-activate in several human cancers including glioblastoma multiforme (GBM), however, the detailed response program of SOX2 in GBM has not yet been defined.

Results: We show that knockdown of the SOX2 gene in LN229 GBM cells reduces cell proliferation and colony formation. We then comprehensively characterize the SOX2 response program by an integrated analysis using several advanced genomic technologies including ChIP-seq, microarray profiling, and microRNA sequencing. Using ChIP-seq technology, we identified 4883 SOX2 binding regions in the GBM cancer genome. SOX2 binding regions contain the consensus sequence wwTGnwTw that occurred 3931 instances in 2312 SOX2 binding regions. Microarray analysis identified 489 genes whose expression altered in response to SOX2 knockdown. Interesting findings include that SOX2 regulates the expression of SOX family proteins SOX1 and SOX18, and that SOX2 down regulates BEX1 (brain expressed X-linked 1) and BEX2 (brain expressed X-linked 2), two genes with tumor suppressor activity in GBM. Using next generation sequencing, we identified 105 precursor microRNAs (corresponding to 95 mature miRNAs) regulated by SOX2, including down regulation of miR-143, $-145,-253-5 p$ and miR-452. We also show that miR-145 and SOX2 form a double negative feedback loop in GBM cells, potentially creating a bistable system in GBM cells.

Conclusions: We present an integrated dataset of ChIP-seq, expression microarrays and microRNA sequencing representing the SOX2 response program in LN229 GBM cells. The insights gained from our integrated analysis further our understanding of the potential actions of SOX2 in carcinogenesis and serves as a useful resource for the research community.
\end{abstract}

\section{Background}

The SOX (SRY-like HMG box) gene family represents a family of transcriptional factors characterized by the presence of a homologous sequence called the HMG (high mobility group) box. The HMG box is a DNA binding domain that is highly conserved throughout eukaryotic species. So far, twenty SOX genes have been identified in humans and mice and they can be divided into 10 subgroups on the basis of sequence similarity and genomic organization $[1,2]$. SOX genes bind to the

\footnotetext{
* Correspondence: greg.foltz@swedish.org; bylin@u.washington.edu

'Swedish Neuroscience Institute, Swedish Medical Center, Seattle, WA 98122, USA

Full list of author information is available at the end of the article
}

minor groove in DNA to control diverse developmental processes [3].

$S O X 2$, one of the key members of the SOX family gene, is highly expressed in embryonic stem cells [4]. Recently, Takahashi et al. showed that SOX2 is a key transcription factor, in conjunction with KLF4, OCT4 and $c-M y c$, whose over expression can induce pluripotency in both mice and human somatic cells $[5,6]$. SOX2 is one of the four factors (OCT4, SOX2, NANOG, and LIN28) that $\mathrm{Yu}$ et al. used to reprogram human somatic cells to pluripotent stem cells that exhibit the essential characteristics of embryonic stem (ES) cells [7]. SOX2 is one of the two factors (SOX2 and OCT4) that were sufficient to generate induced pluripotent stem cells from
C Biomed Central

() 2011 Fang et al; licensee BioMed Central Ltd. This is an Open Access article distributed under the terms of the Creative Commons Attribution License (http://creativecommons.org/licenses/by/2.0), which permits unrestricted use, distribution, and reproduction in any medium, provided the original work is properly cited. 
human cord blood cells [8]. Due to its importance in conferring stemness of cells, the target genes for SOX2 in mouse embryonic stem cells were defined using ChIP-seq technology [9].

SOX2 has also been implicated in several cancers including gastric cancer $[10,11]$, breast cancer $[12,13]$, pancreatic cancer [14], pulmonary non-small cell and neuroendocrine carcinomas [15]. In addition, SOX2 was identified to be a prognostic marker for human esophageal squamous cell carcinoma [16] and rectal cancer [17]. Schmitz et al. found that $S O X 2$ is over expressed in malignant glioma while displaying minimal expression in normal tissues [18]. More recently, Gangemi et al. showed that silencing of the SOX2 in freshly derived glioblastoma tumor-initiating cells (TICs) stopped proliferation and the resulting cells lost tumorigenicity in immunodeficient mice [19]. Ikushima et al. showed that inhibition of TGF-beta signaling drastically deprived tumorigenicity of glioma-initiating cells (GICs) by promoting their differentiation, and that these effects were attenuated in GICs transduced with SOX2 or SOX4 [20]. Taking together, these data suggested that $S O X 2$ is also a key gene in maintaining the stemness of glioma stem cells.

Given that SOX2 is predominantly expressed in embryonic and adult stems cells, including neural progenitor cells, and re-activates in cancers, including malignant gliomas, we hypothesized that the re-activation program of SOX2 may play an important role in the carcinogenesis and maintenance of GBM. Although the $S O X 2$ response program in mouse stem cells was previously defined [9], the re-activation program in cancers such as GBM has not yet been defined. Using ChIP-seq technology, we conducted a genome-wide target identification for SOX2 binding in GBM cells. We generated mRNA expression profiles using the Applied Biosystems' microarray platform and microRNA expression profiles using next-generation sequencing after knockdown of SOX2 expression in GBM cells. An integrated analysis of these data reveals key response programs that potentially play important roles in GBM.

\section{Results}

\section{SOX2 affects colony formation and cell proliferation in} GBM

We previously completed massively parallel signature sequencing (MPSS) and identified SOX2 as significantly over expressed in GBM tissues compared to normal brain tissues [21]. We identified two MPSS tags that correspond to different polyadenylated isoform, and both are up-regulated in GBM tissues compared to normal brain tissues [21]. Our data is consistent with the observation that SOX2 is widely expressed in gliomas including glioblastomas but not in normal brains except for in ependymal layers [22].
To assess the functional consequences of $S O X 2$, we knocked down the $S O X 2$ gene by siRNAs in the GBM cell line LN229 using SOX2 SiRNAs (Ambion Inc.). As shown in Figure 1A, we were able to knockdown SOX2 almost completely using either of the pre-designed SOX2 siRNAs (s13295 and s13296) from Ambion Inc. (Now Applied Biosystems Inc.).

Knockdown of the SOX2 gene in LN229 cells significantly reduced the numbers of colonies formed as shown in Figure 1B. In three replicate experiments, the colony numbers for the MOCK-knockdown cells were 53.3 (STDEV $=2.5$ ) while that for the SOX2 knockdown were 24.7 (STDEV $=2.5)$ ( $\mathrm{T}$-test $\mathrm{P}=0.00015,2$ tails, type 2). Furthermore, knockdown of SOX2 in LN229 cells reduced the numbers of cells, reaching statistical significance at day four (T-test $\mathrm{P}<0.001$ ) and further at day six ( $\mathrm{T}$-test $\mathrm{P}=1.45 \mathrm{E}-06$ ) by MTT assays (Figure $1 \mathrm{C}$ ).

\section{Global identification of SOX2 binding sites in GBM cells by ChIP-seq analysis}

In order to understand the genome-wide binding patterns of $S O X 2$, we applied ChIP-seq technology, which is a novel approach for identifying transcription factor binding sites genome-wide [23,24]. We performed replicate SOX2 ChIP and IgG ChIP. After sequencing analysis, we obtained a total of $1,139,535$ and 638,279 sequence tags respectively for SOX2 and IgG that can be mapped uniquely to the human genome allowing two mismatches.

Using the SISSRs (Site Identification from Short Sequence Reads) ChIP-seq analysis program [25], we identified a total of 4,883 SOX2 binding regions with a $\mathrm{P}$ value $<0.01$ using IgG control ChIP-seq data as the negative control (Additional File 1). We randomly picked 15 genes for which the promoter regions are enriched for the SOX2 IP, and we were able to confirm all 15 genes to be enriched in the SOX2 IP DNAs compared to the IgG-IP DNAs using real time quantitative PCR (Figure 2A), suggesting that the false positive rate is negligible in our dataset.

There are 4714 SOX2 binding regions that can be mapped to TSS (transcription start site) of 3420 known genes. We calculated the distance of the SOX2 binding regions to TSS (transcription start sites) and then tabulated the frequency across the distance intervals before TSS and after TSS. Figure 2B shows that the peak of the SOX2 binding regions is around the TSS sites. We found that about 13\% SOX2 (605 of 4714) binding regions are mapped within $8 \mathrm{~kb}$ of TSS (Figure 2B), and about 25\% (1161 binding regions) are mapped $>8 \mathrm{~kb} \mathrm{5}$ distal to the TSS. The rest mapped to $>8 \mathrm{~kb}$ downstream of TSS start sites of genes.

To understand the function of the $S O X 2$ binding genes, we performed a $\mathrm{GO}$ analysis using the $\mathrm{GO}$ miner 
A:

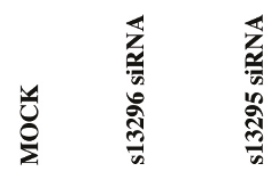

SOX2

B:

\section{SOX2-KO MOCK-control}
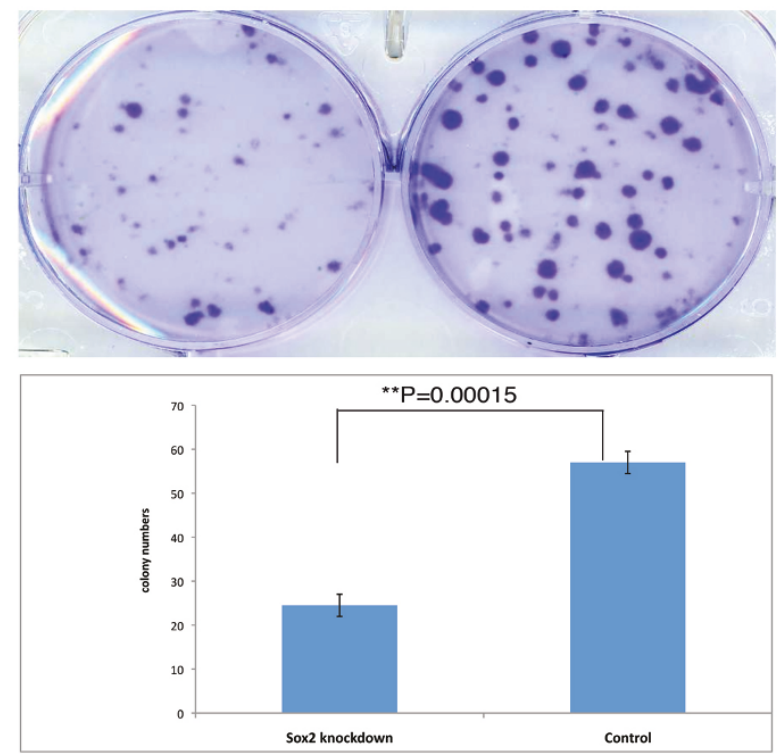

C:

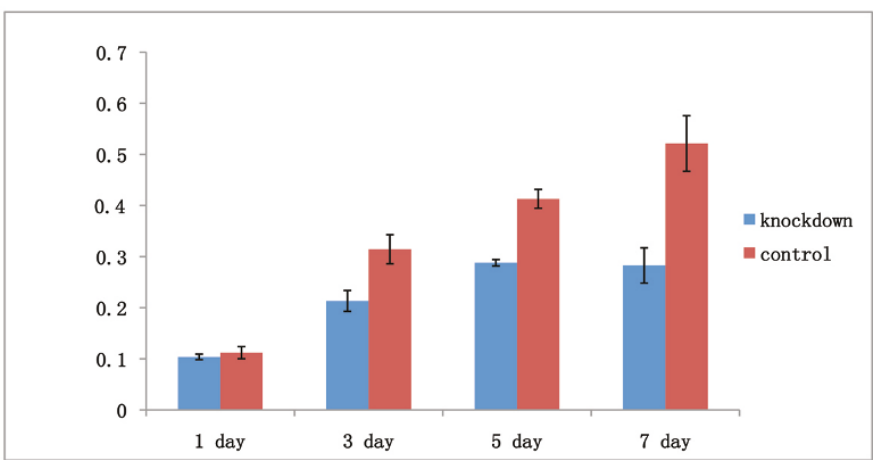

Figure 1 Functional analysis of SOX2 in GBM cells. (A) Western blot analysis showing the known down efficiency of SOX2 siRNAs (s13295 and s13296). (B) Colony formation assays for SOX2 knockdown and mock control. (C). Cell proliferation assays for SOX2 knockdown and mock control.

program using evidence level 3 of molecular functions. The enriched GO terms are shown in Table 1. The top enriched GO terms include GO:0005096 GTPase activator activity, GO:0022843 voltage-gated cation channel activity, GO:0008066 glutamate receptor activity, GO:0005070 SH3 SH2 adaptor activity, GO:0005001 transmembrane receptor protein tyrosine phosphatase activity (Table 1). It should be pointed that expression 
A:

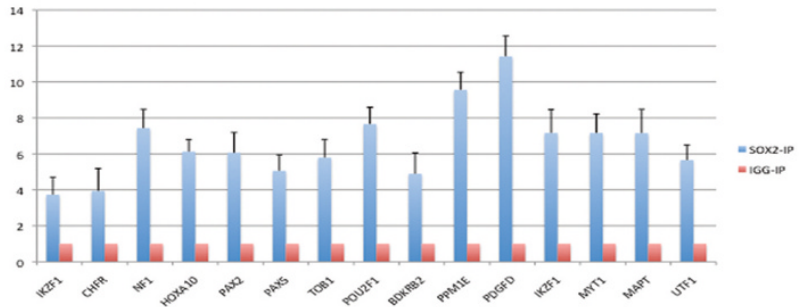

B:

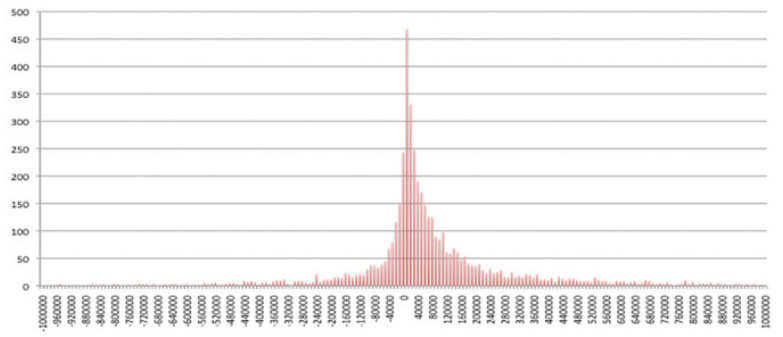

C:

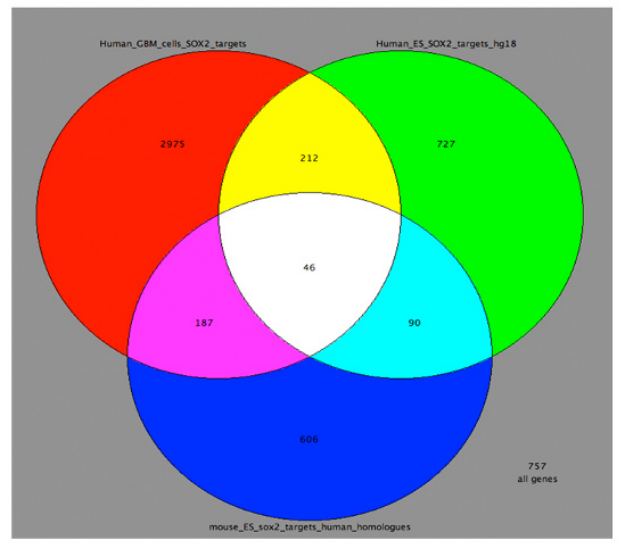

D:

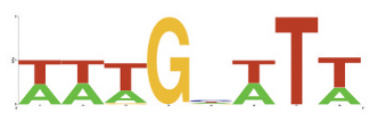

Figure 2 ChIP-seq analysis of SOX2 in GBM cells. Quantitative real time PCR for the confirmation of ChIP-seq peaks. Relative amount of PCR products from SOX2-ChIP and IgG-ChIP were shown as bar graph with the amount of IgG-ChIP normalized to 1. Standard deviations were also shown for SOX2-IP. (B) Histograms of SOX2 binding sites around annotated TSS (Transcription start sites) Frequencies of SOX2 island binding were calculated every 10 kilobases (Y-axis). Relative distance to TSS is shown in X-axis, Negative and positive values indicate localization $5^{\prime}$ or $3^{\prime}$ to TSS respectively. (C). A VENN diagram showing the overlaps of SOX2 targets among human GBM cells, human ES cells and mouse ES cells. (D). The consensus sequence wwTGnwTw with log-likelihood score of 13920.71 identified by the MotifSampler program. The over represented sequences were used as input for the Weblog program (http://weblogo.berkeley.edu/) [53] to display the consensus sequence graphically. 
Table $1 \mathrm{GO}$ terms that are enriched in SOX2 binding genes identified by ChIP-seq

\begin{tabular}{|c|c|c|c|c|c|}
\hline GO CATEGORY & $\begin{array}{l}\text { TOTAL } \\
\text { GENES }\end{array}$ & $\begin{array}{c}\text { Changed } \\
\text { Genes }\end{array}$ & $\begin{array}{l}\text { Enrichment } \\
\text { folds }\end{array}$ & $\begin{array}{l}\text { P value } \\
\text { (Log10) }\end{array}$ & FDR \\
\hline GO:0005096_GTPase_activator_activity & 60 & 27 & 2.39 & -5.54 & 0.01 \\
\hline GO:0004871_signal_transducer_activity & 792 & 196 & 1.31 & -5.17 & 0.003333 \\
\hline GO:0060089_molecular_transducer_activity & 792 & 196 & 1.31 & -5.17 & 0.003333 \\
\hline GO:0060090_molecular_adaptor_activity & 48 & 20 & 2.21 & -3.69 & 0.0175 \\
\hline GO:0022843_voltage-gated_cation_channel_activity & 66 & 25 & 2.01 & -3.67 & 0.014 \\
\hline GO:0008066_glutamate_receptor_activity & 20 & 11 & 2.92 & -3.50 & 0.016667 \\
\hline GO:0004872_receptor_activity & 562 & 137 & 1.29 & -3.41 & 0.02 \\
\hline GO:0005070_SH3_SH2_adaptor_activity & 43 & 18 & 2.22 & -3.40 & 0.02 \\
\hline GO:0004672_protein_kinase_activity & 278 & 75 & 1.43 & -3.37 & 0.017778 \\
\hline GO:0005001_transmembrane_receptor_protein_tyrosine_phosphatase_activity & 18 & 10 & 2.95 & -3.27 & 0.019091 \\
\hline GO:0019198_transmembrane_receptor_protein_phosphatase_activity & 18 & 10 & 2.95 & -3.27 & 0.019091 \\
\hline GO:0005099_Ras_GTPase_activator_activity & 28 & 13 & 2.47 & -3.11 & 0.023333 \\
\hline GO:0030695_GTPase_regulator_activity & 124 & 38 & 1.63 & -3.02 & 0.023077 \\
\hline GO:0016301_kinase_activity & 365 & 92 & 1.34 & -2.92 & 0.027143 \\
\hline GO:0042805_actinin_binding & 4 & 4 & & & \\
\hline GO:0008092_cytoskeletal_protein_binding & 164 & 47 & 1.52 & -2.89 & 0.026667 \\
\hline GO:0016773_phosphotransferase_activity_alcohol_group_as_acceptor & 326 & 83 & 1.35 & -2.82 & 0.029375 \\
\hline GO:0004888_transmembrane_receptor_activity & 410 & 101 & 1.31 & -2.80 & 0.029412 \\
\hline GO:0005244_voltage-gated_ion_channel_activity & 76 & 25 & 1.75 & -2.63 & 0.035263 \\
\hline GO:0022832_voltage-gated_channel_activity & 76 & 25 & 1.75 & -2.63 & 0.035263 \\
\hline GO:0030674_protein_binding_bridging & 58 & 20 & 1.83 & -2.47 & 0.0455 \\
\hline
\end{tabular}

of 196 of 792 genes with signal transducer activity, (GO:0004871), 137 of 562 receptor genes (GO:0004872) (among them, 101 of 410 transmembrane receptor genes, GO: 0004888), and 92 of 365 kinase genes (GO:0016301) (Table 1) was altered in response to SOX2 knockdown.

Marson et al. and Chen et al. recently used ChIP-seq to map binding sites of SOX2 and other key TFs in the mouse ES cells $[9,26]$. Morsen et al. identified 4,087 SOX2 binding sites corresponding to 2,884 genes based on the criteria that a binding site is within $50 \mathrm{~kb}$ of the TSS or TES (transcript end site). Chen et al. identified 4,526 SOX2 binding regions (from their Supplementary Table 3 ) that could be assigned to 2,601 genes of the gene using the same criteria. The union of the two lists generated 4,380 genes. Interestingly, the overlapped genes between the two lists is 1105 genes (25.2\%) (Additional File 2). The difference could be due to the use of different antibodies, Chen et al. used the SOX2 antibody (sc-17320, Santa Cruz Inc) while Morson et al. used an affinity purified goat polyclonal antibody (AF2018, R\&D Systems), or differences in the analysis pipeline and down stream analysis procedures $[9,26]$.

Using the homologene table for human and mouse from NCBI (http://www.ncbi.nlm.nih.gov/homologene), we compared the SOX2 targets that we identified in
LN229 cells with the SOX2 targets that were identified in mouse ES cells [26]. We were able to identify 929 human homologues of 1105 mouse SOX2 binding genes from Chen et al's paper, and then were able to identify 233 unique genes (25\%) (Additional File 1) that are common to the $S O X 2$ binding gene in the human GBM cells (Figure. 2C). These suggest that there are common sets of genes regulated by SOX2 in humans and mice, and in ES cells and in cancer cells. However, we identified many $S O X 2$ binding sites that are only present in the glioblastoma cell line, suggesting that SOX2 targets different pathways in the context of cancer cells.

Boyer et al. applied ChIP-chip technology to identify OCT4, SOX2, and NANOG target genes in human embryonic stem cells using a human promoter array [27]. They identified 1,271 of the SOX2 binding promoter regions for known protein-coding genes in human ES cells. In LN229 cells, we found 258 unique genes that overlapped with their data (Additional File 1 and Figure. 2C). Analysis with the Fisher's Exact test (one sided) revealed that the overlap is highly significant $(\mathrm{P}<$ 0.001). This suggests that while there is some conservation of the genes regulated by $S O X 2$ in ES cells and GBM cells, there are also differences in $S O X 2$ binding regions between the cells. The difference could be due to several factors. First, there are differences in technologies used. The array designed by Boyer et al. covered 
the $-8 \mathrm{~kb}$ to $+2 \mathrm{~kb}$ region relative to each transcription start site of 18,002 transcription start sites representing 17,917 unique genes. If a SOX2 binding region is outside of the region covered by the printed oligos, or is not on the array, it would be missed by ChIP-chip analysis. However, the ChIP-seq technology is not limited by the probes selected to be printed on a chip, and therefore could identify $S O X$ binding regions further upstream or down stream of genes. Second, the $S O X 2$ response program could be different in different cells (i.e. GBM vs. ES cells). It is possible that different SOX proteins interact selectively with and regulate a unique repertoire of target genes, and the selectivity is dependent on the type of cell in which the protein is expressed.

To see whether they were unique functional classification or over representation for the SOX2 targets in GBM cells versus those in human ES cells, we compared 3162 unique SOX2 targets in GBM cells with 817 unique $S O X 2$ targets in human ES cells using GSEA to identify unique over represented GO terms in each set of targets. We found that the unique $S O X 2$ targets in GBM cells were enriched for ion transport, receptor activities, neuron differentiation neurogenesis, etc. (Additional File 3), while the unique SOX2 targets in human ES cells are enriched for macromolecular complex, ion homeostasis, apoptotic program, ATPase activity, etc. (Additional File 4).

We were interested to see whether the genes related to stemness and/or differentiation are SOX2 targets in GBM cells. Using the molecular signature database (http:// www.broadinstitute.org/gsea/msigdb/index.jsp) at the Broad Institute, we found that GO term GO:0045595 (REGULATION OF CELL DIFFERENTIATION) consists of a compiled set of 59 genes related to differentiation. In addition, Ben-Porath et al. curated a gene set with 378 genes over expressed in human embryonic stem cells according to 5 or more out of 20 profiling studies in the Table S1 of their published paper [28]. We found that 17 of 59 genes related to regulation of cell differentiation were SOX2 targets in GBM cells including ACIN1 (apoptotic chromatin condensation inducer 1), BMPR1B (bone morphogenetic protein receptor, type IB), ETS1 (V-ets erythroblastosis virus E26 oncogene homolog 1), SHH (sonic hedgehog homolog, Drosophila), IGFBP3 (insulin-like growth factor binding protein 3) and RUNX1 (Runt-related transcription factor 1) (Additional File 5). In addition, 71 of 378 ES enriched genes were SOX2 targets in GBM cells including CDC2O (cell division cycle 20 homolog of $S$. cerevisiae), CHEK2 (CHK2 checkpoint homolog of S. pombe), FGF13 (fibroblast growth factor 13), RFC3 [replication factor $C$ (activator 1) 3, $38 \mathrm{kDa}$ ] and UTF1 (undifferentiated embryonic cell transcription factor 1) (Additional File 6). However, we did not find OCT4 and NANOG to be SOX2 targets in GBM cells.

\section{Identification of the DNA binding consensus and other known TF binding sites in the SOX2 bound regions}

To see whether the human SOX2 binding regions in GBM cells have their own unique and enriched binding motif, we used the MotifSampler program (http://bioinformatics.psb.ugent.be/webtools/plantcare/html/Motif_Sampler.html) to identify binding consensus sequences enriched in the SOX2 binding regions that we identified. We found a consensus sequence wwTGnwTw with a very high log-likelihood score of 13920.71 . The output matrix for this consensus sequence is shown in Additional File 7, and there are 3931 instances of this motif in 2312 SOX2 binding regions (Additional File 8). The consensus logo is shown in Figure 2D.

We were curious whether known TFs could bind to the $S O X 2$ binding regions that we identified and act as $S O X 2$ cooperators for the regulation of gene expression. In order to systematically search for potential bindings of other transcription factors, we used the MotifScanner program (http://bioinformatics.psb.ugent.be/webtools/ plantcare/html/Motif_Sampler.html) and scanned all TF motif matrices (PWM databases) using the human transcription factor subset of the Transfac professional 7.0. Matched matrices with likelihood (LR) ratios of 500 or higher were tabulated and frequencies calculated (Additional File 9). Among the top known TF matrices (Table 2) that were identified as co-occurred more than $10 \%$ of the time with SOX2 in the SOX2-binding region are: the OCT family, the FOX family, the HNF family, the GATA family and several other TF IRF1 (interferon regulatory factor 1), POU1F1 (POU class 1 homeobox 1), TEF1 (TEAD1, TEA domain family member 1), AREB6 (ZEB1, zinc finger E-box binding homeobox 1) and $G R$ (glucocorticoid receptor, also named NR3C1, nuclear receptor subfamily 3 , group $C$, member 1 ).

POU1F1 is the POU class 1 homeobox 1 . OCT family TFs also contain POU domains. These suggest that SOX2 and many POU domain proteins may act together to control gene expression. SOX2 and OCT family TFs such as OCT1 (POU2F1, POU class 2 homeobox 1) and OCT3/4 (POU5F1, POU class 5 homeobox 1) are well known to work synergistically in embryonic stem cells $[29,30]$. Additionally, we identified novel transcriptional factors in the SOX2 bound regions, including FOX (fork head transcription factor) and HNF (hepatocyte nuclear factor) family proteins. However, its significance remains to be determined. Interestingly, HNF1 (hepatocyte nuclear factor 1) also contains a POU-homeodomain, while HNF3 alpha, which is also named FOXA1, contains a fork head domain [31]. 
Table 2 Top known TFs binding sites in the SOX2 binding regions

\begin{tabular}{lcc}
\hline Known TFs & Number of occurrencies & Percentage of total sites \\
\hline AREB6_04 & 543 & 11.12 \\
FOX_Q2 & 596 & 12.21 \\
FOXD3_01 & 654 & 13.39 \\
FOXJ2_01 & 506 & 10.36 \\
FOXO1_01 & 519 & 10.63 \\
GATA_C & 524 & 10.73 \\
GATA_Q6 & 647 & 13.25 \\
GATA1_04 & 497 & 10.18 \\
GR_Q6_01 & 491 & 10.06 \\
HFH3_01 & 525 & 10.75 \\
HNF1_Q6 & 530 & 10.85 \\
HNF3_Q6 & 528 & 10.81 \\
HNF3ALPHA_Q6 & 649 & 13.29 \\
IRF1_Q6 & 702 & 14.38 \\
OCT_Q6 & 594 & 12.16 \\
OCT1_04 & 697 & 14.27 \\
OCT1_06 & 554 & 11.35 \\
OCT1_Q5_01 & 616 & 12.62 \\
PIT1_Q6 & 571 & 11.69 \\
POU1F1_Q6 & 633 & 12.96 \\
TEF1_Q6 & 776 & 15.89 \\
\hline
\end{tabular}

Microarray analysis reveals that SOX2 knockdown reduces the expression of other SOX family members but up-regulates $B E X 1$ and $B E X 2$

We performed microarray analysis comparing SOX2 knockdown and MOCK transfected LN229 cells, and we identified a total of 565 probes ( 489 known genes with annotations) that were changed $>2$ fold between SOX2 knockdown and SOX2-MOCK transfected LN229 cells (Additional File 10). Array analysis confirmed that SOX2 expression was indeed decreased after SOX2 knockdown. Additionally, we confirmed the array data by RT-PCR analysis of 13 randomly selected up-regulated genes after SOX2 knockdown (BIRC3, NGFR, IL8, CRISPD2, CEBPA, NFKB2, MAP3K14, NFKBIE, NR1H3, APIP, SOCS2, PDGFRA, KIT, BEX1, BEX2, IL-6) (Figure. 3A).

Gene Ontology analysis of all GO categories revealed that proteins belonging to these cellular locations GO:0005576 extracellular region and GO:0005796 Golgi lumen are enriched, and that proteins involved in the GO:0042035 regulation of cytokine biosynthetic process (Table 3). Indeed, we found that knockdown of SOX2 increased the expression of several cytokines including $I L 6$ and IL8,IL23, IL24 and IL32 (Table 4), and the expression of two interleukin receptors-interleukin 7 receptor and interleukin 1 receptor-like 1 . We also found many interesting families of proteins that were regulated by $S O X 2$ by visual inspection of the gene list.
For example, we noticed that two other SOX family protein, SOX1 and SOX18, also exhibited reduced expression by more than 3 fold after SOX2 knockdown (Table 4). We also found that many members of the protocadherins including protocadherin 9,10 , beta 11 , and gamma A3 and C 3 were reduced after SOX2 knockdown. However, the expression of protocadherin alpha 4 was increased after SOX2 knockdown (Table 4). Protocadherin are a large family of cadherin-related molecules that are highly expressed in the brain and their expression appears to be developmentally regulated [32].

The expression of many interesting gene families were up regulated after $S O X 2$ knockdown. For example, we found that brain expressed genes BASP1 (brain abundant, membrane attached signal protein 1 ), $B E X 1$ (brain expressed X-linked 1) and BEX2 (brain expressed X-linked 2) were up regulated after SOX2 knockdown (Table 4). We also found that knocking down $S O X 2$ also increase the expression of many solute carrier family proteins including $S L C 2 A 3$, SLC3A2, SLC7A1, SLC14A1, SLC22A1 and SLC30A1. These solute carrier proteins transport many important solutes such as urea, glucose, organic cations, dibasic and neutral amino acids, zinc, and cationic amino acids (Table 4).

The gene regulated by SOX 2 could be directly regulated or indirectly regulated. By integrating the array data with the ChIP-seq data, the directly targeted genes of SOX2 can be inferred. We found 88 SOX2regulated genes whose promoters were bound by 127 SOX2 binding regions (Figure. 3D and Additional File 1). Interesting genes include $B C L 2$ (B-cell CLL/lymphoma 2) and $B C L 2$ interacting protein 3 (BNIP3), four brain and neuron expressed genes BASP1 (brain abundant, membrane attached signal protein 1), NEDD4 (Neural precursor cell expressed, developmentally down-regulated 4), NRG1 (Neuregulin 1) and NEGR1 (Neuronal growth regulator 1), two interleukins $I L 6$ and $I L 8$, two protocadherins $P C D H 9$ and $P C D H 10$, RUNX1 (Runtrelated transcription factor 1 , acute myeloid leukemia 1 oncogene), and three solute carrier proteins $S L C 3 A 2$, SLC7A1 and SLC30A1 (Additional File 11). The VENN diagram also showed that 11 genes were common SOX2 targets for GBM and ES cells and changed in expression after SOX2 knockdown (Figure. 3D and Table 5). They include EBF3 (early B-cell factor 3), $B A S P 1$ (brain abundant, membrane attached signal protein 1), SLC30A1 (solute carrier family 30 (zinc transporter), member 1), and SLC3A2 (solute carrier family 3 , member 2 ). We speculate that these genes may be involved in GBM stem cells. However, further experimentations are necessary to understand the role of these genes in glioma stem cells. 


\section{A:}

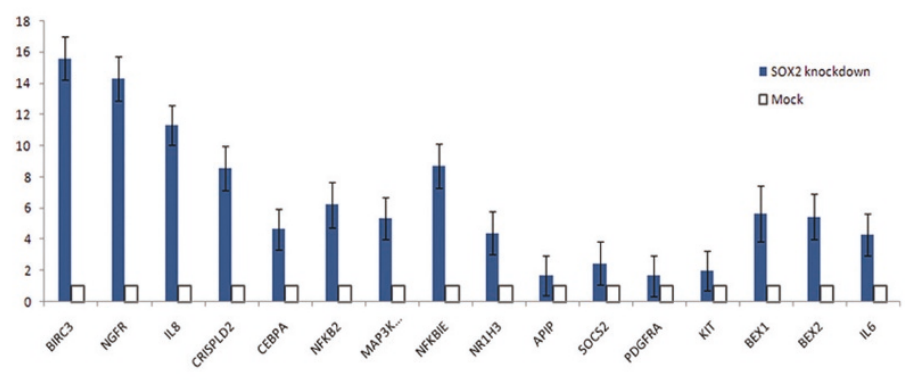

B:

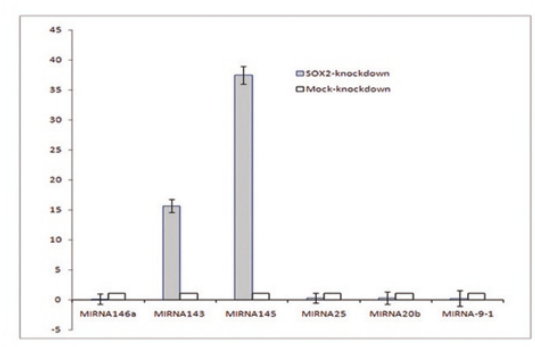

C:

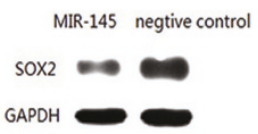

D:

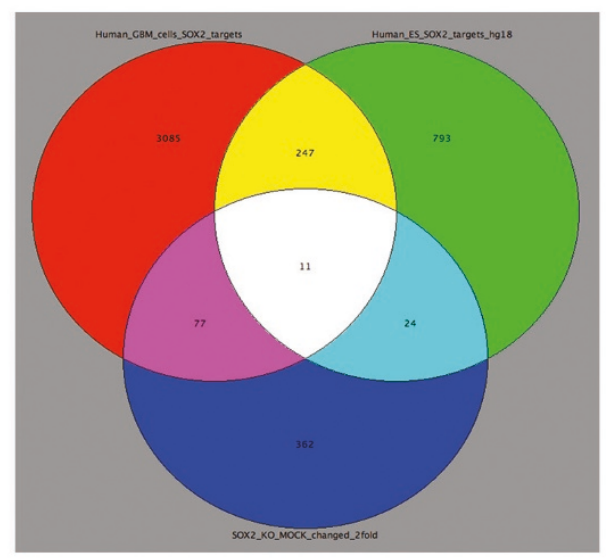

Figure 3 Confirmation of SOX2 regulated genes and microRNAs. (A) Bar graph showing PCR confirmation of the array data of SOX2 gene expression. SOX2-KO: SOX2 knockdown; MOCK, mock known down with negative control siRNAs. Y-axis, relative expression after normalizing the MOCK control to value of 1 ; X-axis, gene symbols. (B). Bar graph showing PCR confirmation of the microRNA sequencing data. Y-axis, relative expression after normalizing the MOCK control to value of 1; X-axis, microRNA names. (C). Western blot analysis showing the SOX2 expression comparing microRNA 145 precursor mimics and a scrambled negative control. (D). A VENN diagram showing the overlaps of SOX2 targets in GBM and ES cells, and the genes that changed expression (2 fold cutoff) after SOX2 knockdown in GBM cells.

\section{SOX2 and miR145 form a double-negative feedback loop} in GBM cells

We also analyzed the effect of SOX2 on miRNAs using next generation sequencing (Illumina). MicroRNA sequencing is an efficient way to identify known and novel microRNAs that are differentially expressed [33].
After miRNA sequencing and data analysis, we found 105 precursor microRNAs (corresponding to 95 mature miRNAs) that were changed $>2$ fold between $S O X 2$ knockdown and SOX2-MOCK transfected LN229 cells (Table 6 and Additional File 12). Six microRNAs including miR-145, -143, $-145^{*},-143^{*},-253-5 \mathrm{p}$ and miR-452, 
Table 3 Enriched GO terms in biological processes for SOX2 DEGs

\begin{tabular}{|c|c|c|c|c|c|}
\hline GO CATEGORY & $\begin{array}{l}\text { TOTAL } \\
\text { GENES }\end{array}$ & $\begin{array}{l}\text { CHANGED } \\
\text { GENES }\end{array}$ & ENRICHMENT & $\begin{array}{c}\text { LOG10 } \\
(p)\end{array}$ & $\begin{array}{c}\text { FALSE DISCOVERY } \\
\text { RATE }\end{array}$ \\
\hline GO:0030935_sheet-forming_collagen & 5 & 4 & 30.33 & -5.64 & 0.00 \\
\hline GO:0042035_regulation_of_cytokine_biosynthetic_process & 43 & 7 & 6.17 & -3.93 & 0.04 \\
\hline GO:0005576_extracellular_region & 561 & 30 & 2.03 & -3.77 & 0.04 \\
\hline GO:0042107_cytokine_metabolic_process & 48 & 7 & 5.53 & -3.62 & 0.05 \\
\hline GO:0002376_immune_system_process & 473 & 26 & 2.08 & -3.51 & 0.05 \\
\hline GO:0042089_cytokine_biosynthetic_process & 47 & 7 & 5.65 & -3.68 & 0.05 \\
\hline GO:0044421_extracellular_region_part & 392 & 24 & 2.32 & -3.98 & 0.06 \\
\hline GO:0006952_defense_response & 320 & 20 & 2.37 & -3.51 & 0.06 \\
\hline GO:0005581_collagen & 24 & 5 & 7.90 & -3.46 & 0.07 \\
\hline GO:0042108_positive_regulation_of_cytokine_biosynthetic_process & 29 & 5 & 6.54 & -3.06 & 0.07 \\
\hline GO:0042226_interleukin-6_biosynthetic_process & 8 & 3 & 14.22 & -3.04 & 0.08 \\
\hline GO:0045408_regulation_of_interleukin-6_biosynthetic_process & 8 & 3 & 14.22 & -3.04 & 0.08 \\
\hline GO:0001525_angiogenesis & 59 & 7 & 4.50 & -3.06 & 0.08 \\
\hline GO:0005796_Golgi_lumen & 7 & 3 & 16.25 & -3.23 & 0.08 \\
\hline GO:0050926_regulation_of_positive_chemotaxis & 7 & 3 & 16.25 & -3.23 & 0.08 \\
\hline GO:0050927_positive_regulation_of_positive_chemotaxis & 7 & 3 & 16.25 & -3.23 & 0.08 \\
\hline GO:0032635_interleukin-6_production & 17 & 4 & 8.92 & -3.07 & 0.08 \\
\hline GO:0032675_regulation_of_interleukin-6_production & 17 & 4 & 8.92 & -3.07 & 0.08 \\
\hline GO:0042445_hormone_metabolic_process & 57 & 7 & 4.66 & -3.15 & 0.08 \\
\hline GO:0044420_extracellular_matrix_part & 46 & 6 & 4.95 & -2.91 & 0.08 \\
\hline GO:0032101_regulation_of_response_to_external_stimulus & 62 & 7 & 4.28 & -2.93 & 0.09 \\
\hline GO:0051093_negative_regulation_of_developmental_process & 271 & 17 & 2.38 & -3.08 & 0.09 \\
\hline GO:0050918_positive_chemotaxis & 9 & 3 & 12.64 & -2.87 & 0.09 \\
\hline GO:0009605_response_to_external_stimulus & 415 & 22 & 2.01 & -2.84 & 0.09 \\
\hline
\end{tabular}

were up regulated after $S O X 2$ knockdown and the rest were down regulated when SOX2 was knocked down. We picked 6 miRNAs to confirm the next generation data using RT-PCR. We confirmed that miR-143 and miR-145 were up regulated after SOX2 knockdown and that miR-146a, -25, -20b and miR-9-1 were down regulated after SOX2 knockdown (Figure. 3B).

$\mathrm{Xu}$ et al. showed that miR-145 targets SOX2 and down regulates its expression in human embryonic stem cells [34]. To see whether the same is true in GBM cells, we transfected LN229 GBM cell with miR-145 mimics and we found that miR-145 also decreased SOX2 expression in GBM cells (Figure. 3C). As knocking down SOX2 up regulates miR145 in the RT-PCR and next-generation sequencing data (Figure. $3 \mathrm{~B}$ and Table 6), this suggests that $S O X 2$ itself down regulates miR-145. Taken together, SOX2 down regulates miR145 and miR145 also down regulates SOX2, suggesting that SOX2 and miR145 form a double-negative feedback loop in GBM cells. We also checked to see whether there are SOX2 binding regions in the proximity of miR145 genomic locus. We found that there are no SOX2 binding regions with significant $\mathrm{P}$ values $(\mathrm{P}<$ 0.01 ) in the close proximity of the miR145 locus. The closest one is about $23 \mathrm{~kb}$ from the miR145 genomic locus. This may suggest that the SOX2 feedback regulation of miR145 is indirect, not resulting from direct binding of the SOX2 to the miR145 genomic region.

\section{Discussion}

We applied ChIP-seq technology to identify global SOX2 binding regions in GBM cells. To our best knowledge, this is the first global analysis of SOX2's binding regions in cancer cells. SOX2 encodes a member of the SRYrelated HMG-box $(S O X)$ family of transcription factors. We investigated SOX2's global binding targets by ChIPSeq analysis, and found that SOX2 binding regions in GBM cells are enriched for AT nucleotides with a consensus sequence wwTGnwTw $[\mathrm{w}=\mathrm{A}$ or $\mathrm{T}$; its reverse and complement strand wAwnCAww]. The mouse sox 2 consensus motif in the mouse ES cells found by Chen et al. has the sequence 5'-CATTGTT-3' [26]. The similarity lies in that both consensus sequences are $\mathrm{AT}$ rich sequences with a core TG di-nucleotide flanked by AT rich sequences. The difference may due to the fact that they derived from different types of cells (ES vs. glioma) and species (human vs. mouse).

The AT rich sequence we identified for SOX2 consensus is consistent with previous in vitro studies showing that the HMG domain of SOX proteins binds to the 
Table 4 Examples of SOX2 regulated genes and families

\begin{tabular}{lcl}
\hline Gene Symbol & ratio SOX2_KO/MOCK & Description \\
\hline SOX family & & \\
SOX1 & 0.32 & SRY (sex determining region Y)-box 1 \\
SOX18 & 0.50 & SRY (sex determining region Y)-box 18 \\
SOX2 & 0.30 & SRY (sex determining region Y)-box 2
\end{tabular}

\section{Brain-expressed}

BASP1 $\quad 3.52$

BEX2 $\quad 3.29$

BEX1 $\quad 4.81$

G protein-coupled receptor

\section{GPR1}

GPR172B

GPR37

\subsection{2}

2.07

0.49

brain abundant, membrane attached signal protein 1

brain expressed $\mathrm{X}$-linked 2

brain expressed, $\mathrm{X}$-linked 1

G protein-coupled receptor 1

$\mathrm{G}$ protein-coupled receptor 172B

$\mathrm{G}$ protein-coupled receptor 37

Interleukins and their receptors

$\begin{array}{lcl}\text { ILIRL1 } & 2.48 & \text { interleukin } 1 \text { receptor-like } 1 \\ \text { IL23A } & 2.60 & \text { interleukin 23, alpha subunit p19 } \\ \text { IL24 } & 5.73 & \text { interleukin } 24 \\ \text { IL32 } & 2.42 & \text { interleukin } 32 \\ \text { IL6 } & 10.25 & \text { interleukin } 6 \text { (interferon, beta 2) } \\ \text { IL7R } & 2.32 & \text { interleukin } 7 \text { receptor } \\ \text { IL8 } & 2.22 & \text { interleukin } 8\end{array}$

Solute carrier family

$\begin{array}{lll}\text { SLC14A1 } & 4.81 & \text { solute carrier family } 14 \text { (urea transporter), member } 1 \text { (Kidd blood group) } \\ \text { SLC2A3 } & 2.29 & \text { solute carrier family } 2 \text { (facilitated glucose transporter), member } 3 \\ \text { SLC22A1 } & 3.80 & \text { solute carrier family } 22 \text { (organic cation transporter), member } 1 \\ \text { SLC3A2 } & 2.07 & \text { solute carrier family } 3 \text { (activators of dibasic and neutral amino acid transport), member } 2 \\ S L C 30 A 1 & 2.62 & \text { solute carrier family } 30 \text { (zinc transporter), member } 1 \\ \text { SLC7A1 } & 2.25 & \text { solute carrier family } 7 \text { (cationic amino acid transporter, y+ system), member } 1\end{array}$

Protocadherin family

$\begin{array}{lll}\text { PCDH10 } & 0.45 & \text { protocadherin } 10 \\ \text { PCDH9 } & 0.32 & \text { protocadherin } 9 \\ \text { PCDHA4 } & 2.20 & \text { protocadherin alpha } 4 \\ \text { PCDHB11 } & 0.38 & \text { protocadherin beta } 11 \\ \text { PCDHGA3 } & 0.49 & \text { protocadherin gamma subfamily A, 3 } \\ \text { PCDHGC3 } & 0.43 & \text { protocadherin gamma subfamily } C, 3\end{array}$

minor groove of DNA through AT rich sequences with a heptamer motif WWCAAAG (W = A or T) $[35,36]$. Therefore we have identified AT rich SOX2 specific binding sequences. Before the development of ChIPchip or ChIP-Seq technologies, Mertin et al. determined the DNA-binding properties of SOX9 using random oligonucleotide selection assay [37] and they identified a core sequence of an AT rich sequence AACAAT or wwCAAw (w, A or T) for SOX9 binding. The HMG domain in SOX family proteins forms an L-shaped module composed of three helices that binds to DNA in the minor groove. SOX proteins are categorized into Groups A-G based on their sequence homology [38]. SOX2 belongs to Group A (also named SRY) and SOX9 belongs to group $\mathrm{E}$ [38]. The amino acid sequence identity of the HMG domain within the same group is high at $>90 \%$, however, the amino acid sequence identity between distant groups decreases to $~ 60 \%$ [38]. A sequence alignment revealed that SOX2 and SOX9 only have about $61 \%$ amino acid sequence identity in the HMG domain. The sequence variations may explain the similar AT rich properties yet different consensus in 
Table 5 Common SOX2 targets of human GBM and ES cells that changed expression after SOX2 knockdown

\begin{tabular}{lcl}
\hline Gene Symbol & Mock/SOX2_KO ratios & Description \\
\hline ALCAM & 2.02 & activated leukocyte cell adhesion molecule \\
BASP1 & 0.28 & brain abundant, membrane attached signal protein 1 \\
BCAT1 & 0.38 & branched chain aminotransferase 1, cytosolic \\
COL12A1 & 2.17 & collagen, type XII, alpha 1 \\
CTH & 0.37 & cystathionase (cystathionine gamma-lyase) \\
EBF3 & 2.03 & early B-cell factor 3 \\
KLF5 & 0.49 & Kruppel-like factor 5 (intestinal) \\
ONECUT1 & 0.48 & one cut domain, family member 1 \\
PTHLH & 0.34 & parathyroid hormone-like hormone \\
SLC3OA1 & 0.38 & solute carrier family 30 (zinc transporter), member 1 \\
SLC3A2 & 0.48 & solute carrier family 3 (activators of dibasic and neutral amino acid transport), member 2 \\
\hline
\end{tabular}

their binding regions for SOX2 and SOX9. Additional functional binding assays including mutagenesis and footprinting analysis will be needed to confirm the binding activities and specificities. Further experimentation is therefore warranted.

It was a surprise to find that about one quarter of genes regulated by SOX2 encompass important GO categories: 196 out of 792 genes (about 25\%) were found to have signal transducer activity (GO:0004871), 101 of 410 belong to transmembrane receptor genes (about 25\%) (GO: 0004888), and 92 of 365 are kinase genes (about 25\%) (GO:0016301). Signal transducer, receptor and kinase genes are important genes that play an essential role in cellular functions and therefore it is not surprising that SOX2 is an essential gene that plays important roles in development and in carcinogenesis.

We found that BEX1 (brain expressed X-linked 1) and $B E X 2$ (brain expressed X-linked 2) were up regulated after SOX2 knockdown (Table 4). We have previously shown that BEX1 and BEX2 are silenced in GBM tumor specimens and exhibited extensive promoter hypermethylation [39]. We demonstrated by in vitro and in a xenograft mouse model that $B E X 1$ or $B E X 2$ possess tumor suppressor activity [39]. Our data suggested that SOX2 might down regulate $B E X 1$ and $B E X 2$ expression, reducing their tumor suppressor activities and thus promoting carcinogenesis. However, we did not find SOX2 binding regions in the $B E X 1$ and $B E X 2$ gene loci, suggesting the down regulation was properly an indirect effect of $S O X 2$ knockdown.

We found that $S O X 2$ also regulates the expression of SOX family protein SOX1 and SOX18 (Table 4). SOX1 plays roles in neural determination and differentiation [40] and is a neural stem cell marker [41]. Bylund et al. showed that sox 1 , sox 2 and sox 3 are the transcription factors that keep neural cells undifferentiated by counteracting the activity of proneural proteins [42]. However, the role of $S O X 1$ in GBM has not yet been studied. SOX18 plays important roles in blood vasculature formation [43].
Young et al. assessed the effects of disrupted SOX18 function on MCF-7 human breast cancer and human umbilical vein endothelial cell (HUVEC) proliferation by measuring BrdU incorporation and by MTT assay, cell migration using Boyden chamber assay, and capillary tube formation in vitro [44]. They showed that over expression of wild-type SOX18 promoted capillary tube formation of HUVECs in vitro, whereas expression of dominant-negative SOX18 impaired tube formation of HUVECs [44]. Therefore, SOX18 is a potential target for antiangiogenic therapy of human cancers. The role of $S O X 18$ in GBM has not been studied. Taking together, $S O X 2$ could act through $S O X 1$ and $S O X 18$, and thus play roles in both maintaining stem cell properties of glioma cells and forming tumor vasculature in gliomas, which are two major obstacles preventing us from treating these tumors effectively.

By microRNA sequencing we determined that levels of 105 precursor microRNAs (corresponding to 95 mature miRNAs) are altered in response to $S O X 2$ knockdown (Table 6 and Additional File 12). We showed that SOX2 could down regulate the expression of miR-143 and miR-145. miR-145 was shown to be down regulated in several cancers such as colon cancers [45] and prostate cancers [46], and miR-143 was shown to be down regulated in colon cancers [47] and bladder cancers [48]. The relationship of miR-143 and miR-145 and GBM has not been studied and is worth future investigation.

We further demonstrated that SOX2 and miR-145 form a double-negative regulation loop in GBM cells (Figure. 4A). Double-negative feedback loop involving microRNAs and their targets have been observed previously [49,50]. A double-negative feedback mechanism has been proposed as a mechanism to form bistability in cellular states. Johnston et al. demonstrated that the stability and irreversibility of the terminal differentiated state of neuronal cells is ensured by a double-negative feedback loop between two microRNAs $l s y-6$ and mir-273 and their transcription factor targets in the nematode Caenorhabditis elegans [50]. 
Table 6 SOX2 regulated miRNAs identified by next generation sequencing

\begin{tabular}{|c|c|c|c|}
\hline $\begin{array}{l}\text { precursor miRNA } \\
\text { ID }\end{array}$ & $\begin{array}{l}\text { mature miRNA } \\
\text { ID }\end{array}$ & $\begin{array}{c}\text { MOCK/SOX2 } \\
\text { KO }\end{array}$ & $P$ value \\
\hline hsa-mir-106a & hsa-miR-106a & 17.89 & $1.94 \mathrm{E}-68$ \\
\hline hsa-mir-106a & hsa-miR-106a* & 20.50 & $2.41 \mathrm{E}-06$ \\
\hline hsa-mir-106b & hsa-miR-106b* & 2.68 & $2.30 \mathrm{E}-05$ \\
\hline hsa-mir-106b & hsa-miR-106b & 2.55 & 9.03E-05 \\
\hline hsa-mir-10b & hsa-miR-10b & 2.70 & 1.09E-05 \\
\hline hsa-mir-125b-1 & hsa-miR-125b & 2.80 & 2.79E-06 \\
\hline hsa-mir-125b-1 & hsa-miR-125b-1* & 2.62 & $6.78 \mathrm{E}-05$ \\
\hline hsa-mir-125b-2 & hsa-miR-125b & 2.80 & $2.75 \mathrm{E}-06$ \\
\hline hsa-mir-1268 & hsa-miR-1268 & 2.85 & 5.28E-06 \\
\hline hsa-mir-1271 & hsa-miR-1271 & 3.44 & 0.000102818 \\
\hline hsa-mir-1287 & hsa-miR-1287 & 6.63 & 6.21E-17 \\
\hline hsa-mir-1301 & hsa-miR-1301 & 3.60 & $5.03 \mathrm{E}-06$ \\
\hline hsa-mir-1305 & hsa-miR-1305 & 3.28 & $2.17 \mathrm{E}-05$ \\
\hline hsa-mir-1307 & hsa-miR-1307 & 4.36 & $6.38 \mathrm{E}-16$ \\
\hline hsa-mir-130b & hsa-miR-130b* & 3.97 & $2.19 \mathrm{E}-11$ \\
\hline hsa-mir-135b & hsa-miR-135b* & 5.26 & 8.40E-19 \\
\hline hsa-mir-135b & hsa-miR-135b & 5.09 & $2.86 \mathrm{E}-17$ \\
\hline hsa-mir-140 & hsa-miR-140-3p & 8.69 & $2.82 \mathrm{E}-41$ \\
\hline hsa-mir-140 & hsa-miR-140-5p & 7.93 & $4.29 E-37$ \\
\hline hsa-mir-143 & hsa-miR-143 & 0.02 & $3.04 \mathrm{E}-182$ \\
\hline hsa-mir-143 & hsa-miR-143* & 0.05 & $2.41 \mathrm{E}-72$ \\
\hline hsa-mir-145 & hsa-miR-145 & 0.02 & $1.99 E-132$ \\
\hline hsa-mir-145 & hsa-miR-145* & 0.03 & $4.74 \mathrm{E}-113$ \\
\hline hsa-mir-146a & hsa-miR-146a & 17.38 & $1.05 \mathrm{E}-73$ \\
\hline hsa-mir-146a & hsa-miR-146a* & 11.40 & $7.59 \mathrm{E}-07$ \\
\hline hsa-mir-149 & hsa-miR-149 & 4.27 & $3.27 E-14$ \\
\hline hsa-mir-15b & hsa-miR-15b* & 3.18 & $3.05 \mathrm{E}-05$ \\
\hline hsa-mir-17 & hsa-miR-17 & 3.28 & 2.85E-09 \\
\hline hsa-mir-187 & hsa-miR-187 & 9.55 & $1.40 \mathrm{E}-09$ \\
\hline hsa-mir-188 & hsa-miR-188-5p & 4.83 & $2.82 \mathrm{E}-08$ \\
\hline hsa-mir-18a & hsa-miR-18a & 3.46 & $1.76 \mathrm{E}-09$ \\
\hline hsa-mir-18b & hsa-miR-18b & 21.77 & $1.55 E-47$ \\
\hline hsa-mir-190 & hsa-miR-190 & 2.78 & $2.00 \mathrm{E}-05$ \\
\hline hsa-mir-196a-1 & hsa-miR-196a & 2.58 & $5.85 \mathrm{E}-05$ \\
\hline hsa-mir-196a-2 & hsa-miR-196a & 2.55 & $9.28 \mathrm{E}-05$ \\
\hline hsa-mir-1977 & hsa-miR-1977 & 0.32 & 4.88E-07 \\
\hline hsa-mir-200c & hsa-miR-200c & 2.63 & $5.38 \mathrm{E}-05$ \\
\hline hsa-mir-204 & hsa-miR-204 & 38.20 & $4.44 \mathrm{E}-83$ \\
\hline hsa-mir-20a & hsa-miR-20a & 3.03 & $1.10 \mathrm{E}-07$ \\
\hline hsa-mir-20b & hsa-miR-20b & 24.84 & $1.62 \mathrm{E}-88$ \\
\hline hsa-mir-20b & hsa-miR-20b* & 16.86 & 4.09E-28 \\
\hline hsa-mir-217 & hsa-miR-217 & 6.54 & $1.10 \mathrm{E}-10$ \\
\hline hsa-mir-224 & hsa-miR-224* & 0.13 & $4.22 \mathrm{E}-18$ \\
\hline hsa-mir-2276 & hsa-miR-2276 & 11.25 & $2.06 \mathrm{E}-15$ \\
\hline hsa-mir-25 & hsa-miR-25 & 2.92 & $5.28 \mathrm{E}-07$ \\
\hline hsa-mir-28 & hsa-miR-28-3p & 2.70 & $1.25 \mathrm{E}-05$ \\
\hline hsa-mir-30b & hsa-miR-30b & 4.91 & $1.20 \mathrm{E}-19$ \\
\hline hsa-mir-30d & hsa-miR-30d & 4.80 & $2.04 \mathrm{E}-19$ \\
\hline hsa-mir-30d & hsa-miR-30d* & 4.79 & 8.38E-12 \\
\hline hsa-mir-320a & hsa-miR-320a & 2.54 & 0.000106251 \\
\hline
\end{tabular}

Table 6 SOX2 regulated miRNAs identified by next generation sequencing (Continued)

hsa-mir-330

hsa-mir-338

hsa-mir-338

hsa-mir-339

hsa-mir-340

hsa-mir-361

hsa-mir-362

hsa-mir-363

hsa-mir-423

hsa-mir-452

hsa-mir-454

hsa-mir-454

hsa-mir-484

hsa-mir-500

hsa-mir-501

hsa-mir-501

hsa-mir-502

hsa-mir-514-1

hsa-mir-514-2

hsa-mir-514-3

hsa-mir-532

hsa-mir-532

hsa-mir-548d-1

hsa-mir-548d-2

hsa-mir-548n

hsa-mir-573

hsa-mir-574

hsa-mir-577

hsa-mir-584

hsa-mir-585

hsa-mir-589

hsa-mir-589

hsa-mir-590

hsa-mir-592

hsa-mir-615

hsa-mir-616

hsa-mir-626

hsa-mir-652

hsa-mir-660

hsa-mir-671

hsa-mir-671

hsa-mir-760

hsa-mir-873

hsa-mir-877

hsa-mir-889

hsa-mir-9-1

hsa-mir-9-1

hsa-mir-9-2

hsa-mir-9-2

hsa-mir-9-3

hsa-mir-9-3
hsa-miR-330-5p

hsa-miR-338-5p

hsa-miR-338-3p

hsa-miR-339-5p

hsa-miR-340*

hsa-miR-361-3p

hsa-miR-362-5p

hsa-miR-363

hsa-miR-423-3p

hsa-miR-452

hsa-miR-454*

hsa-miR-454

hsa-miR-484

hsa-miR-500*

hsa-miR-501-3p

hsa-miR-501-5p

hsa-miR-502-3p

hsa-miR-514

hsa-miR-514

hsa-miR-514

hsa-miR-532-5p

hsa-miR-532-3p

hsa-miR-548d-5p

hsa-miR-548d-5p

hsa-miR-548n

hsa-miR-573

hsa-miR-574-5p

hsa-miR-577

hsa-miR-584

hsa-miR-585

hsa-miR-589*

hsa-miR-589

hsa-miR-590-3p

hsa-miR-592

hsa-miR-615-3p

hsa-miR-616

hsa-miR-626

hsa-miR-652

hsa-miR-660

hsa-miR-671-5p

hsa-miR-671-3p

hsa-miR-760

hsa-miR-873

hsa-miR-877

hsa-miR-889

hsa-miR-9

hsa-miR-9*

hsa-miR-9

hsa-miR-9*

hsa-miR-9

hsa-miR-9*
3.90

42.70

32.33

2.75

4.53

2.66

4.32

21.08

3.19

0.20

7.09

2.79

3.32

4.18

6.15

5.17

4.19

9.25

9.25

9.25

4.74

3.72

3.52

3.45

3.84

5.67

4.68

14.88

9.12

46.00

3.76

2.83

3.15

24.45

3.79

3.26

0.12

3.99

4.27

2.73

2.83

10.74

11.50

4.22

0.16

10.74

11.87

10.74

11.87

10.74

11.06
5.23E-06

2.78E-75

6.56E-64

4.52E-05

5.95E-10

6.29E-05

8.19E-13

2.70E-82

1.05E-08

4.36E-59

8.55E-13

7.68E-05

1.74E-08

2.46E-14

2.77E-19

6.25E-11

9.80E-11

0.000108265

0.000108265

0.000108265

2.71E-18

1.47E-07

2.17E-08

3.03E-08

2.66E-08

6.41E-06

8.73E-18

6.79E-60

1.41E-42

2.35E-14

3.23E-08

2.95E-06

6.09E-06

1.71E-52

5.88E-09

6.21E-05

2.40E-10

1.57E-12

1.17E-10

8.59E-06

5.90E-05

1.39E-27

1.21E-14

2.51E-13

3.89E-05

2.37E-50

3.43E-35

2.39E-50

3.43E-35

2.37E-50

2.66E-35 
Table 6 SOX2 regulated miRNAs identified by next generation sequencing (Continued)

\begin{tabular}{llll}
\hline hsa-mir-92a-1 & hsa-miR-92a & 3.38 & $5.65 \mathrm{E}-10$ \\
hsa-mir-92a-2 & hsa-miR-92a & 3.35 & $8.85 \mathrm{E}-10$ \\
hsa-mir-93 & hsa-miR-93 & 3.46 & $1.43 \mathrm{E}-10$ \\
hsa-mir-942 & hsa-miR-942 & 3.43 & $3.82 \mathrm{E}-07$ \\
\hline
\end{tabular}

A positive-feedback loop or double-negative feedback loops can convert graded inputs into switch-like, irreversible responses [51]. Such a system will be "bistable" as the system exists almost exclusively in one of two possible states. Bistability has been shown in several signal transduction and transcriptional regulatory events such as the p42 mitogen-activated protein kinase and c-Jun aminoterminal kinase pathways in Xenopus oocytes [51]. For a bistable system with two components $\mathrm{A}$ and $\mathrm{B}$, the system will toggle between two stable states: one with $\mathrm{A}$ on and $\mathrm{B}$ off and one with B on and A off. For example, for the SOX2 and miR-145 bistable system, the system can be on SOX2 on, miR-145 off state or SOX2 off, miR-145 on state (Figure. 4B). Further experimentation will be necessary to analyze in detail the two cellular states for the SOX2miR145 double-negative feedback loop in GBM cells.

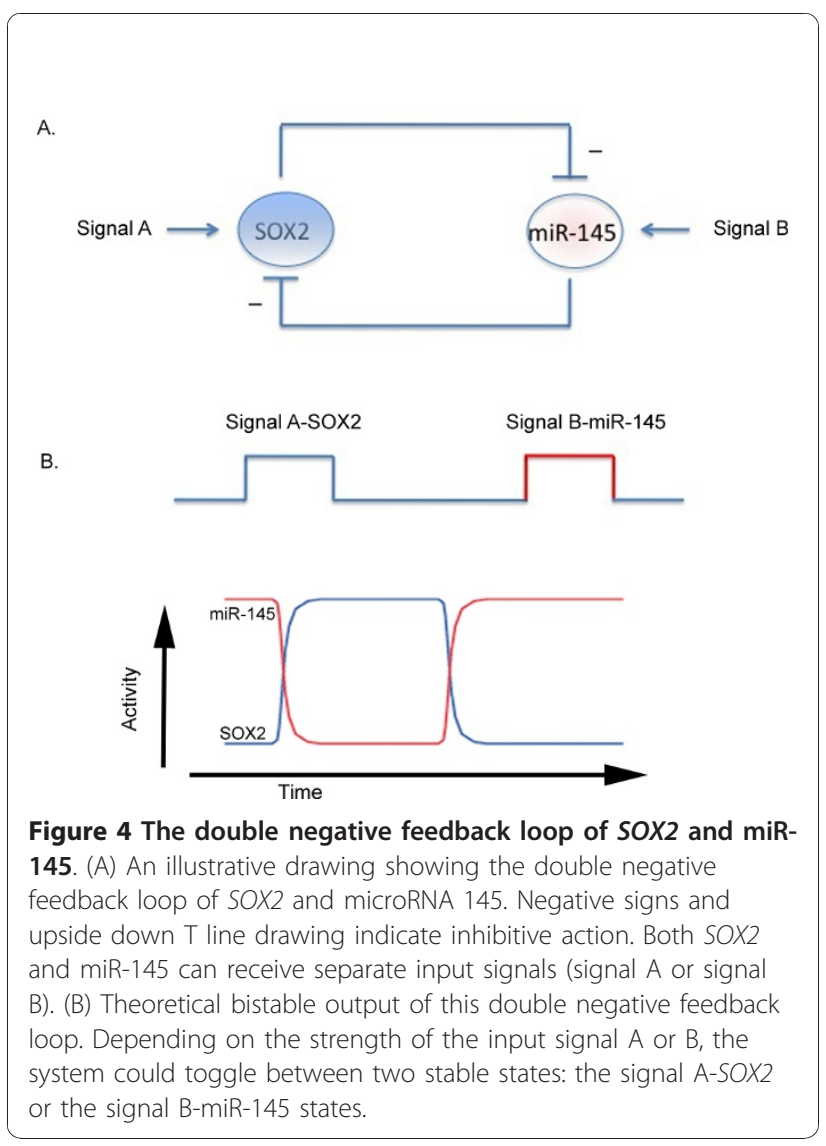

\section{Conclusion}

We have comprehensively characterized the SOX2 response program by integrated analysis using several advanced technologies including ChIP-seq, microarrays and microRNA sequencing. The datasets of ChIP-seq, microarrays and microRNA sequencing of SOX2 response program, which, to our best knowledge, are the first datasets of $S O X 2$ in cancers, will be useful resources for the research community. Furthermore, the insights we gained from our integrated analysis further our understanding of the roles of SOX2 in carcinogenesis.

\section{Methods}

\section{Cell culture and functional assays}

LN229 cells were obtained from the American Type Culture Collection (Manassas, VA) and maintained in DMEM with $10 \%$ fetal bovine serum. Cell proliferation was analyzed using the MTT assay kits (Millipore, Billerica, MA) according to the manufacturer's protocol. For Soft agar colony formation assay, cells were trypsinized and counted. 10,000 cells were seeded in six-well plates. After 2 weeks of growth, colonies with a diameter greater than $4 \mathrm{~mm}$ were counted. Experiments were performed in quadruplicates [39].

\section{Chromatin immunoprecipition (ChIP) - Sequencing}

About $3 \times 10^{6}$ LN229 cells were used for chromatin immunoprecipition (ChIP) assay, carried out according to the manufacturer's instructions (Millipore, EZ-Magna ChIP $^{\text {max }}$ A). Antibodies used for ChIP included SOX2 (ab59776, Abcam Inc.) and IgG (sc-2027, Santa Cruz Biotehnology Inc.). For ChIP, SOX2 antibody was tested for its specificity and specific band was found (Figure 1A). ChIP assay was performed using the ChIP kit (Upstate Biotechnology, Lake Placid, NY) according to the manufacturer's protocol. Briefly, cells were crosslinked by adding fresh formaldehyde to cell culture medium to a final concentration of $1 \%$. Fixation was monitored at $37^{\circ} \mathrm{C}$ for $10 \mathrm{~min}$. The fixed cells were resuspended in the lysis buffer. Nuclei were collected by centrifugation at $2000 \times g$, and resuspended in the nuclei lysis buffer. Samples were sonicated on ice to the length of 200-500 base pairs. $5 \mu \mathrm{g}$ antibody and $50 \mu \mathrm{l}$ Dynal protein $G$ beads were incubated for 2 hours at $4^{\circ} \mathrm{C}$. Sonicated chromatin were incubated with the protein G-antibody complex overnight at $4^{\circ} \mathrm{C}$. Precipitated immunocomplex was treated with proteinase $\mathrm{K}$ for 2 hours at $65^{\circ} \mathrm{C}$, and DNA was purified Qiagen Qiaquick PCR purification kit. ChIP DNA end repairing, adaptor ligation, and amplification were performed as described earlier [23]. Fragments of about 200 bp (without linkers) were isolated from agarose gel and used for sequencing using the Illumina $2 \mathrm{G}$ genetic analyzer. Illumina data analysis pipeline was performed as described [23]. 
For this manuscript, the same genome build hg18 and its associated annotations were used for all analysis. Sequence reads that map to multiple sites in the human genome were removed. To identify SOX2 binding peaks, we used SISSRs (Site Identification from Short Sequence Reads) (http://www.rajajothi.com/sissrs/) [25] with default parameters with E-value is set to 10 , P value set to 0.001. SOX2 ChIP-seq as positive and IgG control ChIP-seq data as negative input.

To calculate the distance to the TSS start site, annotations from the UCSC (hg18) were used. We also took into consideration the direction of the strand when we calculated the distance to TSS. As the SOX2 binding regions is always recorded on the positive strands, for genes mapped to the positive strand, the distance is the end position of the SOX2 binding region minus the TSS start position; for genes mapped to the negative strand, the distance is the TSS start position minus the SOX2 binding region start position.

\section{Validation of ChIP-seq datasets by ChIP-qPCR}

We selected a list of binding peaks for validation using quantitative real-time PCR. The primers were listed in the Additional File 13. Three replicates were run. Realtime PCR was performed using the SYBR ${ }^{\circledR}$ Green (Invitrogen) dye detection method on ABI PRISM 7900 HT Sequence Detection System under default conditions: $95^{\circ} \mathrm{C}$ for $10 \mathrm{~min}$, and 35 cycles of $95^{\circ} \mathrm{C}$ for $15 \mathrm{~s}$ and $55^{\circ} \mathrm{C}$ for $1 \mathrm{~min}$. Comparative $\mathrm{C}_{\mathrm{t}}$ method was used for quantification of the transcripts.

\section{Gene Ontology Analysis}

High-Throughput GoMiner [52] was used to find statistically over represented Gene Ontology (GO) terms. GO terms of all evidence levels and categories were used for the analysis. The algorithm used by the High-throughput GoMiner [52], which is the one-sided Fisher exact p value corrected for multiple comparisons, was used to calculate the FDR (false discovery rate). To identify over represented GO terms in the 3162 unique SOX2 targets in GBM cells versus the 817 unique SOX2 targets in human ES cells, we GSEA (http://www.broadinstitute. org/gsea/index.jsp). The parameters used were: 1000 permutations using the C5 gene sets (GO gene sets), the diff_of_classes algorithm as metric for ranking genes, weighted enrichment statistic, minimum gene set size of 3. Other parameters were set as default.

\section{Motif scanning and identification}

To identify novel motifs, SOX2 binding regions identified by ChIP-seq were extended to $100 \mathrm{bp} \mathrm{5}$ ' and 3' and the sequences were retrieved in FASTA format. The sequences were first subjected to the RepeatMask program (http://www.repeatmasker.org/) to mask all human repeats. We used the MotifSampler to find overexpressed motifs in the $S O X 2$ binding regions with the default parameters. The over represented sequences were used as input for the Weblog program (http:// weblogo.berkeley.edu/) [53] to display the consensus sequence graphically. For a systematic search for all potential transcription binding sites, we used the Motifscanner software (http://bioinformatics.psb.ugent.be/ webtools/plantcare/html/Motif_Sampler.html). Human upstream sequences from EPD (The Eukaryotic Promoter Database) (epd_homo_sapiens_499_chromgenes_non_split_3.bg) were downloaded from the motif scanner web site and used as the background model. The human subset of the Transfac professional 7.0 PWM matrices was used. Matched TF matrices with likelihood ratios (LR) of 500 or higher were tabulated and their frequencies calculated.

\section{Small interfering RNA transfection}

SOX2 SiRNAs (Ambion Inc) were used for transient knockdown of SOX2. The SiRNA sequences are: s13295, sense sequence AGUGGAAACUUUUGUCGGATT and antisense sequence, UCCGACAAAAGUUUCCACUCG; S13296, sense sequence ACCAGCGCAUGGACAGUUATT and anti-sense sequence UAACUGUCCAUGCGCUGGUTC. LN229 cells were seeded into six well plates, cultured overnight and transfected with SOX2 SiRNAs at a final concentration of $100 \mathrm{nM}$ using TransIT-OT1 (Mirus Bio LLC, Madison WI) according to the manufacturer's instructions. At 72 hours after tansfection, cells were harvested for western blot analysis and for microarray analysis.

\section{Microarray analysis}

The Applied Biosystems' microarray platform was used using the standard array hybridization protocol as we described previously [39]. The ABI arrays contain 31,700 60-mer oligonucleotide probes representing 29,098 individual human genes. Two biological replicates were performed including cell transfection and microarray analysis. GeneSpring program were used to analyze the array data. The raw signal intensities individual probes were combined (averaged) based on Celera's Gene ID (ABI's annotation table), and then imported into the GeneSpring program, and data from individual chip were normalized per chip with $75 \%$ percentile and normalized per gene by median. To filter out those lowly expressed genes across all chips, only arrays data with a signal to noise $(\mathrm{S} / \mathrm{N})$ ratio of $>3$ in one of the arms (SOX2 Knockdown or mock knockdown) were used for analysis. Two biological replicates were performed. The normalized data for the replicates were averaged for each gene. To identify differentially expressed genes, a two fold cutoff value was used. 


\section{Integrative analysis}

The human genome build hg18 and its associated annotations were used for all analysis. The SOX2 binding regions identified by ChIP-seq (Table S2) was annotated with the genome annotation of hg18 for their associated genes using the nearest gene within $50 \mathrm{~kb}$ of the TSS (transcription start site) or TES (transcription end site). As the published human ChIP-Chip data in human embryonic stem cells [27] was annotated in 2005 with human genome hg17 when the paper was published, the annotation was outdated. We first converted the SOX2 binding regions coordinates (Table S3 of the Boyer's paper) into the hg18 coordinates using the tool liftover (http://genome.ucsc. edu/cgi-bin/hgLiftOver). We re-annotated the human ES SOX2 binding regions with hg18 annotations using the chromosomal coordinates with the same criteria as we used for the GBM SOX2 ChIP-seq data. There were 1,075 human ES SOX2 binding regions that could be annotated with nearby genes. To identify overlapping genes between SOX2 ChIP-seq and the microarray data, we used the gene symbols from the HUGO gene nomenclature committee (http://www.genenames.org/) to compare the two lists. To compare the human ChIP-seq data with the sox 2 targets that were identified in mouse ES cells [26], we used the homologene table for human and mouse from NCBI (http://www.ncbi.nlm.nih.gov/homologene) to identify the human homologues for the mouse sox 2 targets and then used the human homologues to compare with the human SOX2 ChIP-seq data.

\section{RNA isolation and Small RNA sequencing}

RNA was isolated from cells using mirVana ${ }^{\mathrm{Tm}}$ miRNA Isolation Kit (Ambion, Austin, TX) according to the manufacturer's instructions. Sequencing library preparation was carried out according to Illumina mirna sample preparation protocol. Small RNA samples were sequenced using GA2 sequencer (Illumina)

For data analyses, Solexa adapters were first trimmed from raw sequences using custom Perl scripts, and the trimmed sequences were then aligned to known human miRNAs precursors (miRBase release 14) using miRExpress [54]. The -t parameter (alignment identity between query and reference sequences) for miRExpress was set to be 0.9 . The expression abundance of corresponding miRNAs were counted by miRExpress and normalized by the counts of trimmed sequences in the library and used for further analysis.

Differentially expressed miRNAs were identified by statistics analysis using edgeR package from bioconductor[55]. The top differentially expressed ones were clustered by $\mathrm{MeV}[56,57]$.

\section{Validation of miRNAs expression by RT-PCR}

We selected a list of miRNAs for validation using quantitative real-time PCR. The primers were available upon request. Three replicates were run. Real-time PCR was performed using the $\mathrm{SYBR}^{\odot}$ Green (Invitrogen) dye detection method on ABI PRISM 7900 HT Sequence Detection System under default conditions: $95^{\circ} \mathrm{C}$ for $30 \mathrm{~s}$, and 40 cycles of $95^{\circ} \mathrm{C}$ for $15 \mathrm{~s}$ and $60^{\circ} \mathrm{C}$ for $20 \mathrm{~s}$. Comparative $\mathrm{Ct}$ method was used for quantification of the transcripts.

\section{Transfection of microRNA}

microRNA-145 precursor mimics was obtained from Ribobio company (Guanzhou China). A scrambled precursor with no homology to the human genome was used as controls. LN229 cells were transfected with the precursor mimics by lipofectamine 2000 (Invitrogen).

\section{Data access}

The array data for SOX 2 knockdown and control data from this study have been submitted to Gene Expression Omnibus (GEO) under accession No.GSE23839. The SOX2 ChIP-seq data from this study have been submitted to Gene Expression Omnibus (GEO) under accession No. GSE23795.

\section{Additional material}

Additional file 1: SOX2 binding regions in GBM cells identified by ChIP-seq.

Additional file 2: Common genes from the two SOX2 Chip_seq datasets from mouse ES cells.

Additional file 3: Over represented GO terms in 3162 unique SOX2 targets in GBM cells

Additional file 4: Over represented GO terms in 817 unique SOX2 targets in human ES cells.

Additional file 5: SOX2 targets that are related to regulation of cell differentiation defined by GO term GO:0045595 (REGULATION OF CELL DIFFERENTIATION).

Additional file 6: SOX2 targets that are related to stemness defined by $\mathbf{3 7 8}$ genes overexpressed in human embryonic stem cells according to 5 or more out of 20 profiling studies in the Table S1 of Ben-Porath et al.'s paper [28].

Additional file 7: The SOX2 consensus matrix

Additional file 8: The frequency of the matches to the SOX2 matrix. Additional file 9: Frequencies of known TFs binding sites cooccurred in the SOX2 binding regions.

Additional file 10: Differentially expressed genes between SOX2Knockdown cells and control cells.

Additional file 11: SOX2 binding and changed in gene expression.

Additional file 12: SOX2 regulated miRNAs identified by Illumina's next generation sequencing.

Additional file 13: Primers for Q-RT-PCR for confirmation of ChIPSeq data. 


\section{Abbreviations}

ChIP-seq: Chromatin immunoprecipitation-Sequencing; SRY: sex determining region Y; ShRNA: small hairpin RNA.

\section{Acknowledgements}

This work was supported by grants 2006AA02A303, 2006AA02Z4A2, 2006DFA32950, 2007DFC30360 and 2004CB518707 from the MOST, China to BL. The work was also support by Swedish Medical Foundation (GF). The authors declare no conflicts of interest.

\section{Author details}

'Swedish Neuroscience Institute, Swedish Medical Center, Seattle, WA 98122, USA. ${ }^{2}$ Zhejiang-California International NanoSystems Institute, Zhejiang University, Hangzhou, Zhejiang 310029, PR China. ${ }^{3}$ Cancer Institute (Key Laboratory of Cancer Prevention and Intervention, China National Ministry of Education), The Second Affiliated Hospital, Zhejiang University School of Medicine, Hangzhou, Zhejiang 310009, PR China. ${ }^{4}$ Department of Urology, University of Washington, Seattle, WA 98195, USA. ${ }^{5}$ Department of Medicinal Chemistry, University of Washington, BOX 367610, Seattle, WA 98195, USA. ${ }^{6}$ The Institute for Systems Biology, Seattle, WA 98103, USA.

\section{Authors' contributions}

$\mathrm{BL}$ and $\mathrm{XF}$ designed this study. XF, JY, LL performed the experiments. WY performed data analysis. SZ, LH, DRG, GF help to design the study and wrote the paper. All authors read and approved the final manuscript.

Received: 31 August 2010 Accepted: 6 January 2011

Published: 6 January 2011

\section{References}

1. Bowles J, Schepers G, Koopman P: Phylogeny of the SOX family of developmental transcription factors based on sequence and structural indicators. Dev Biol 2000, 227(2):239-255.

2. Schepers GE, Teasdale RD, Koopman P: Twenty pairs of sox: extent, homology, and nomenclature of the mouse and human sox transcription factor gene families. Dev Cell 2002, 3(2):167-170.

3. Wegner M: From head to toes: the multiple facets of Sox proteins. Nucleic Acids Res 1999, 27(6):1409-1420.

4. Avilion AA, Nicolis SK, Pevny LH, Perez L, Vivian N, Lovell-Badge R: Multipotent cell lineages in early mouse development depend on SOX2 function. Genes Dev 2003, 17(1):126-140.

5. Takahashi K, Yamanaka S: Induction of pluripotent stem cells from mouse embryonic and adult fibroblast cultures by defined factors. Cell 2006, 126(4):663-676.

6. Takahashi K, Tanabe K, Ohnuki M, Narita M, Ichisaka T, Tomoda K, Yamanaka S: Induction of pluripotent stem cells from adult human fibroblasts by defined factors. Cell 2007, 131(5):861-872.

7. Yu J, Vodyanik MA, Smuga-Otto K, Antosiewicz-Bourget J, Frane JL, Tian S, Nie J, Jonsdottir GA, Ruotti V, Stewart R, et al: Induced pluripotent stem cell lines derived from human somatic cells. Science 2007, 318(5858):1917-1920.

8. Giorgetti A, Montserrat N, Rodriguez-Piza I, Azqueta C, Veiga A, Izpisua Belmonte JC: Generation of induced pluripotent stem cells from human cord blood cells with only two factors: Oct4 and Sox2. Nat Protoc 5(4):811-820.

9. Marson A, Levine SS, Cole MF, Frampton GM, Brambrink T, Johnstone S, Guenther MG, Johnston WK, Wernig M, Newman J, et al: Connecting microRNA genes to the core transcriptional regulatory circuitry of embryonic stem cells. Cell 2008, 134(3):521-533.

10. Li XL, Eishi Y, Bai YQ, Sakai H, Akiyama Y, Tani M, Takizawa T, Koike M, Yuasa $Y$ : Expression of the SRY-related HMG box protein SOX2 in human gastric carcinoma. Int J Oncol 2004, 24(2):257-263.

11. Park ET, Gum JR, Kakar S, Kwon SW, Deng G, Kim YS: Aberrant expression of SOX2 upregulates MUC5AC gastric foveolar mucin in mucinous cancers of the colorectum and related lesions. Int J Cancer 2008, 122(6):1253-1260.

12. Rodriguez-Pinilla SM, Sarrio D, Moreno-Bueno G, Rodriguez-Gil Y, Martinez MA, Hernandez L, Hardisson D, Reis-Filho JS, Palacios J: Sox2: a possible driver of the basal-like phenotype in sporadic breast cancer. Mod Pathol 2007, 20(4):474-481.
13. Chen Y, Shi L, Zhang L, Li R, Liang J, Yu W, Sun L, Yang X, Wang Y, Zhang $Y$, et al: The molecular mechanism governing the oncogenic potential of SOX2 in breast cancer. J Biol Chem 2008, 283(26):17969-17978.

14. Sanada Y, Yoshida K, Ohara M, Oeda M, Konishi K, Tsutani Y: Histopathologic evaluation of stepwise progression of pancreatic carcinoma with immunohistochemical analysis of gastric epithelial transcription factor SOX2: comparison of expression patterns between invasive components and cancerous or nonneoplastic intraductal components. Pancreas 2006, 32(2):164-170.

15. Sholl LM, Long KB, Hornick JL: Sox2 Expression in Pulmonary Non-small Cell and Neuroendocrine Carcinomas. Appl Immunohistochem Mol Morphol 2009.

16. Wang Q, He W, Lu C, Wang Z, Wang J, Giercksky KE, Nesland JM, Suo Z Oct3/4 and Sox 2 are significantly associated with an unfavorable clinical outcome in human esophageal squamous cell carcinoma. Anticancer Res 2009, 29(4):1233-1241.

17. Saigusa $S$, Tanaka $K$, Toiyama $Y$, Yokoe $T$, Okugawa $Y$, loue $Y$, Miki C, Kusunoki M: Correlation of CD133, OCT4, and SOX2 in Rectal Cancer and Their Association with Distant Recurrence After Chemoradiotherapy. Ann Surg Oncol 2009.

18. Schmitz M, Temme A, Senner V, Ebner R, Schwind S, Stevanovic S, Wehner R, Schackert G, Schackert HK, Fussel M, et al: Identification of SOX2 as a novel glioma-associated antigen and potential target for $\mathrm{T}$ cellbased immunotherapy. Br J Cancer 2007, 96(8):1293-1301.

19. Gangemi RM, Griffero F, Marubbi D, Perera M, Capra MC, Malatesta P, Ravetti GL, Zona GL, Daga A, Corte G: SOX2 silencing in glioblastoma tumor-initiating cells causes stop of proliferation and loss of tumorigenicity. Stem Cells 2009, 27(1):40-48.

20. Ikushima H, Todo T, Ino Y, Takahashi M, Miyazawa K, Miyazono K: Autocrine TGF-beta signaling maintains tumorigenicity of glioma-initiating cells through Sry-related HMG-box factors. Cell Stem Cell 2009, 5(5):504-514.

21. Lin B, Madan A, Yoon JG, Fang X, Yan X, Kim TK, Hwang D, Hood L, Foltz G: Massively parallel signature sequencing and bioinformatics analysis identifies up-regulation of TGFBI and SOX4 in human glioblastoma. PLOS One 2010, 5(4):e10210.

22. Phi JH, Park SH, Kim SK, Paek SH, Kim JH, Lee YJ, Cho BK, Park CK, Lee DH, Wang KC: Sox2 expression in brain tumors: a reflection of the neuroglial differentiation pathway. Am J Surg Pathol 2008, 32(1):103-112.

23. Barski A, Cuddapah S, Cui K, Roh TY, Schones DE, Wang Z, Wei G, Chepelev I, Zhao K: High-resolution profiling of histone methylations in the human genome. Cell 2007, 129(4):823-837.

24. Johnson DS, Mortazavi A, Myers RM, Wold B: Genome-wide mapping of in vivo protein-DNA interactions. Science 2007, 316(5830):1497-1502.

25. Jothi R, Cuddapah S, Barski A, Cui K, Zhao K: Genome-wide identification of in vivo protein-DNA binding sites from ChIP-Seq data. Nucleic Acids Res 2008, 36(16):5221-5231.

26. Chen $X, X u H$, Yuan $P$, Fang F, Huss $M$, Vega VB, Wong E, Orlov YL, Zhang $W$, Jiang J, et al: Integration of external signaling pathways with the core transcriptional network in embryonic stem cells. Cell 2008, 133(6):1106-1117.

27. Boyer LA, Lee TI, Cole MF, Johnstone SE, Levine SS, Zucker JP, Guenther MG, Kumar RM, Murray HL, Jenner RG, et al: Core transcriptional regulatory circuitry in human embryonic stem cells. Cell 2005, 122(6):947-956.

28. Ben-Porath I, Thomson MW, Carey VJ, Ge R, Bell GW, Regev A, Weinberg RA: An embryonic stem cell-like gene expression signature in poorly differentiated aggressive human tumors. Nat Genet 2008, 40(5):499-507.

29. Okumura-Nakanishi S, Saito M, Niwa H, Ishikawa F: Oct-3/4 and Sox2 regulate Oct-3/4 gene in embryonic stem cells. J Biol Chem 2005 , 280(7):5307-5317.

30. Williams DC Jr, Cai M, Clore GM: Molecular basis for synergistic transcriptional activation by Oct1 and Sox2 revealed from the solution structure of the 42-kDa Oct1.Sox2.Hoxb1-DNA ternary transcription factor complex. J Biol Chem 2004, 279(2):1449-1457.

31. Costa RH, Kalinichenko W, Holterman AX, Wang X: Transcription factors in liver development, differentiation, and regeneration. Hepatology 2003, 38(6):1331-1347.

32. Sano K, Tanihara H, Heimark RL, Obata S, Davidson M, St John T, Taketani S, Suzuki S: Protocadherins: a large family of cadherin-related molecules in central nervous system. EMBO J 1993, 12(6):2249-2256. 
33. Morin RD, Zhao Y, Prabhu AL, Dhalla N, McDonald H, Pandoh P, Tam A, Zeng T, Hirst M, Marra M: Preparation and analysis of microRNA libraries using the Illumina massively parallel sequencing technology. Methods Mol Biol 650:173-199.

34. Xu N, Papagiannakopoulos T, Pan G, Thomson JA, Kosik KS: MicroRNA-145 regulates OCT4, SOX2, and KLF4 and represses pluripotency in human embryonic stem cells. Cell 2009, 137(4):647-658.

35. Harley VR, Jackson DI, Hextall PJ, Hawkins JR, Berkovitz GD, Sockanathan S, Lovell-Badge R, Goodfellow PN: DNA binding activity of recombinant SRY from normal males and XY females. Science 1992, 255(5043):453-456.

36. van de Wetering $M$, Clevers $H$ : Sequence-specific interaction of the HMG box proteins TCF-1 and SRY occurs within the minor groove of a Watson-Crick double helix. EMBO J 1992, 11(8):3039-3044.

37. Mertin S, McDowall SG, Harley VR: The DNA-binding specificity of SOX9 and other SOX proteins. Nucleic Acids Res 1999, 27(5):1359-1364.

38. Kamachi $Y$, Uchikawa M, Kondoh H: Pairing SOX off: with partners in the regulation of embryonic development. Trends Genet 2000, 16(4):182-187.

39. Foltz G, Ryu GY, Yoon JG, Nelson T, Fahey J, Frakes A, Lee H, Field L, Zander K, Sibenaller Z, et al: Genome-wide analysis of epigenetic silencing identifies BEX1 and BEX2 as candidate tumor suppressor genes in malignant glioma. Cancer Res 2006, 66(13):6665-6674.

40. Pevny LH, Sockanathan S, Placzek M, Lovell-Badge R: A role for SOX1 in neural determination. Development 1998, 125(10):1967-1978.

41. Alcock J, Sottile V: Dynamic distribution and stem cell characteristics of Sox1-expressing cells in the cerebellar cortex. Cell Res 2009, 19(12):1324-1333.

42. Bylund $M$, Andersson E, Novitch BG, Muhr J: Vertebrate neurogenesis is counteracted by Sox1-3 activity. Nat Neurosci 2003, 6(11):1162-1168.

43. Francois M, Caprini A, Hosking B, Orsenigo F, Wilhelm D, Browne C, Paavonen K, Karnezis T, Shayan R, Downes M, et al: Sox18 induces development of the lymphatic vasculature in mice. Nature 2008, 456(7222):643-647.

44. Young N, Hahn CN, Poh A, Dong C, Wilhelm D, Olsson J, Muscat GE, Parsons P, Gamble JR, Koopman P: Effect of disrupted SOX18 transcription factor function on tumor growth, vascularization, and endothelial development. J Natl Cancer Inst 2006, 98(15):1060-1067.

45. Arndt GM, Dossey L, Cullen LM, Lai A, Druker R, Eisbacher M, Zhang C, Tran N, Fan H, Retzlaff $K$, et al: Characterization of global microRNA expression reveals oncogenic potential of miR-145 in metastatic colorectal cancer. BMC Cancer 2009, 9:374.

46. Zaman MS, Chen Y, Deng G, Shahryari V, Suh SO, Saini S, Majid S, Liu J, Khatri G, Tanaka $Y$, et al: The functional significance of microRNA-145 in prostate cancer. Br J Cancer 103(2):256-264.

47. Akao $Y$, Nakagawa $Y$, Naoe T: MicroRNA-143 and -145 in colon cancer. DNA Cell Biol 2007, 26(5):311-320.

48. Lin T, Dong W, Huang J, Pan Q, Fan X, Zhang C, Huang L: MicroRNA-143 as a tumor suppressor for bladder cancer. J Urol 2009, 181(3):1372-1380.

49. Bracken CP, Gregory PA, Kolesnikoff N, Bert AG, Wang J, Shannon MF, Goodall GJ: A double-negative feedback loop between ZEB1-SIP1 and the microRNA-200 family regulates epithelial-mesenchymal transition. Cancer Res 2008, 68(19):7846-7854.

50. Johnston RJ Jr, Chang S, Etchberger JF, Ortiz CO, Hobert O: MicroRNAs acting in a double-negative feedback loop to control a neuronal cell fate decision. Proc Natl Acad Sci USA 2005, 102(35):12449-12454.

51. Ferrell JE Jr: Self-perpetuating states in signal transduction: positive feedback, double-negative feedback and bistability. Curr Opin Cell Biol 2002, 14(2):140-148.

52. Zeeberg BR, Qin H, Narasimhan S, Sunshine M, Cao H, Kane DW, Reimers M, Stephens RM, Bryant D, Burt SK, et al: High-Throughput GoMiner, an 'industrial-strength' integrative gene ontology tool for interpretation of multiple-microarray experiments, with application to studies of Common Variable Immune Deficiency (CVID). BMC Bioinformatics 2005, 6:168.

53. Crooks GE, Hon G, Chandonia JM, Brenner SE: WebLogo: a sequence logo generator. Genome Res 2004, 14(6):1188-1190.

54. Wang WC, Lin FM, Chang WC, Lin KY, Huang HD, Lin NS: miRExpress: analyzing high-throughput sequencing data for profiling microRNA expression. BMC Bioinformatics 2009, 10:328.

55. Robinson MD, McCarthy DJ, Smyth GK: edgeR: a Bioconductor package for differential expression analysis of digital gene expression data. Bioinformatics 2010, 26(1):139-140.
56. Saeed Al, Sharov V, White J, Li J, Liang W, Bhagabati N, Braisted J, Klapa M, Currier T, Thiagarajan M, et al: TM4: a free, open-source system for microarray data management and analysis. Biotechniques 2003, 34(2):374-378.

57. Saeed Al, Bhagabati NK, Braisted JC, Liang W, Sharov V, Howe EA, Li J, Thiagarajan M, White JA, Quackenbush J: TM4 microarray software suite. Methods Enzymol 2006, 411:134-193.

doi:10.1186/1471-2164-12-11

Cite this article as: Fang et al:: The SOX2 response program in

glioblastoma multiforme: an integrated ChIP-seq, expression microarray, and microRNA analysis. BMC Genomics 2011 12:11.

\section{Submit your next manuscript to BioMed Central and take full advantage of:}

- Convenient online submission

- Thorough peer review

- No space constraints or color figure charges

- Immediate publication on acceptance

- Inclusion in PubMed, CAS, Scopus and Google Scholar

- Research which is freely available for redistribution

Submit your manuscript at www.biomedcentral.com/submit
C Biomed Central 